TITLE:

\title{
Evaluation of the potential of doxorubicin loaded microbubbles as a theranostic modality using a murine tumor model.
}

\section{AUTHOR(S):}

Abdalkader, Rodi; Kawakami, Shigeru; Unga, Johan; Suzuki, Ryo; Maruyama, Kazuo; Yamashita, Fumiyoshi; Hashida, Mitsuru

\section{CITATION:}

Abdalkader, Rodi ...[et al]. Evaluation of the potential of doxorubicin loaded microbubbles as a theranostic modality using a murine tumor model.. Acta biomaterialia 2015, 19: 112118

\section{ISSUE DATE:}

2015-03-17

URL:

http://hdl.handle.net/2433/198724

\section{RIGHT:}

(c) 2015 Acta Materialia Inc. Licensed under the Creative Commons Attribution-NonCommercial-NoDerivatives 4.0 International http://creativecommons.org/licenses/by-nc-nd/4.0/. NOTICE: this is the author's version of a work that was accepted for publication in [Acta Biomaterialia]. Changes resulting from the publishing process, such as peer review, editing, corrections, structural formatting, and other quality control mechanisms may not be reflected in this document. Changes may have been made to this work since it was submitted for publication. A definitive version was subsequently published in [Acta Biomaterialia, Volume 19, Pages 112-118] doi:10.1016/j.actbio.2015.03.014; 許諾条件に より本文ファイルは2017-03-17に公開.; This is not the published version. Please cite only the published version.; この論 文は出版社版でありません。引用の際には出版社版をご確認ご利用ください。 


\section{Acta Biomaterialia}

\section{Evaluation of the potential of doxorubicin loaded microbubbles as a theranostic} modality using a murine tumor model

Rodi Abdalkader ${ }^{1}$, Shigeru Kawakami ${ }^{2, *}$, Johan Unga ${ }^{1}$, Ryo Suzuki ${ }^{3}$, Kazuo Maruyama ${ }^{3}$ Fumiyoshi Yamashita ${ }^{1}$, and Mitsuru Hashida ${ }^{1,4, *}$

${ }^{1}$ Kyoto University, Graduate School of Pharmaceutical Sciences, Kyoto, Japan

${ }^{2}$ Nagasaki University, Graduate School of Biomedical Sciences, Nagasaki, Japan

${ }^{3}$ Laboratory of Drug and Gene Delivery, Faculty of Pharma-sciences, Teikyo University, Japan

${ }^{4}$ Kyoto University, Institute for Integrated Cell-Material Science (iCeMS), Kyoto, Japan

*Corresponding authors. Tel. \& Fax: +81757534575 (M. Hashida), +81 958192450 (S. Kawakami).

E-mail addresses: skawakam@nagasaki-u.ac.jp (S. Kawakami)

hashidam@pharm.kyoto-u.ac.jp (M. Hashida) 


\section{Graphical abstract}

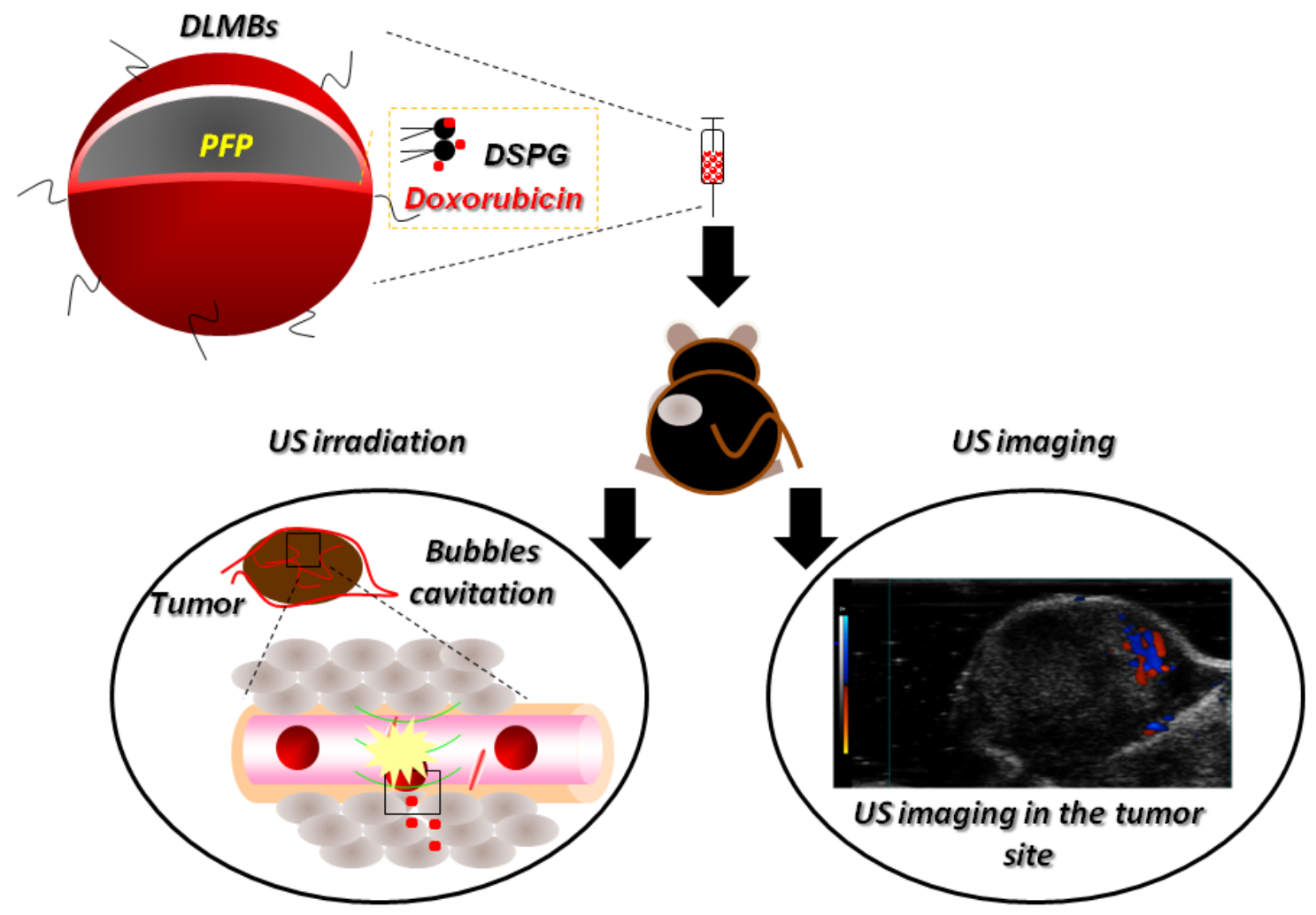




\section{Abstract}

In this study, a novel phospholipid-based microbubble formulation containing doxorubicin and perfluoropropane gas (DLMB) was developed. The DLMBs were prepared by mechanical agitation of a phospholipid dispersion in the presence of perfluoropropane (PFP) gas. An anionic phospholipid, distearoylphosphatidylglycerol (DSPG) was selected to load doxorubicin in the microbubbles by means of electrostatic interaction. The particle size, zeta potential, echogenicity and stability of the DLMBs were measured. Drug loading was $\geq 92 \%$. The potential of the DLMBs for use as a theranostic modality were evaluated in tumor bearing mice. Gas chromatography analysis of PFP showed significant enhancement of PFP retention when doxorubicin was used at concentrations of $10-82 \%$ equivalent to DSPG. The inhibitory effects on the proliferation of B16BL6 melanoma murine cells in vitro were enhanced using a combination of ultrasound (US) irradiation and DLMBs. Moreover, in vivo DLMBs in combination with (US) irradiation significantly inhibited the growth of B16BL6 melanoma tumor in mice. Additionally, US echo imaging showed high contrast enhancement of the DLMBs in the tumor vasculature. These results suggest that DLMBs could serve as US triggered carriers of doxorubicin as well as tumor imaging agents in cancer therapy.

Key words: Cancer therapy, Doxorubicin loaded microbubbles, Theranostics, Ultrasound, Cavitation 


\section{Introduction}

In cancer therapy, targeted drug delivery to the tumor is a great challenge [1,2]. Many recent studies have shown that microbubbles (MBs) serve as a potential modality for both tumor therapy and imaging [3]. When systemically administrated, the interaction between the perfluoropropane (PFP) gas in MBs and an ultrasound (US) wave result in contrast signal enhancement in the blood vessels. However, irradiating MBs with higher pressure amplitude US causes cavitations [4,5]. These effects, known as sonoporation, enhance the delivery of drugs and nucleic acids into cells for improving therapeutic outcome [6]. Although US is a safe, easily performed, and relatively cheap technology, US imaging lacks high resolution especially in the case of deep tissues. Therefore, the formulation allows us not only to determine MB distribution but also to achieve treatment response at the tumor site in a noninvasive manner $[7,8]$.

Doxorubicin is one of the most frequently used anti-cancer agents for a variety of solid tumors. Combination of doxorubicin loaded MBs (DLMBs) with US irradiation has been found to enhance doxorubicin uptake through US induced cavitations effects $[9,10]$. Regarding the stability of doxorubicin encapsulation into liposomes, the use of anionic phospholipids may be suitable for loading via electrostatic interaction $[11,12]$. For theranostic applications concerning the tumor, not only doxorubicin but also PFP gas should be stably encapsulated into the formulation. However, MBs lack stability under in vivo conditions as a result of gas diffusion and leakage $[8,13]$.

For the stable encapsulation of PFP gas into bubble formulations, the selection of lipid is an important factor $[14,15]$. So far, our group has developed positively charged bubble liposomes for gene or siRNA delivery in combination with US irradiation $[16,17]$. Additionally, we have developed negatively charged bubble lipopolyplex using 1,2- 
distearoyl-sn-glycero-3-phosphatidylglycerol (DSPG) as an anionic lipid for safe and efficient transfection by diminished interaction with erythrocytes [18,19]. Recently, Sax et al. have reported that DSPG incorporated bubble (acoustic) liposomes had an enhanced halflife both in vitro and in vivo [20]. Taking all of these factors into consideration, we expect that the use of anionic MBs partly containing DSPG can offer a platform for the development of more stable DLMBs with theranostic applications in cancer therapy.

In the present study, we optimized the conditions required for the preparation of stable DLMBs using negatively charged MBs in relation to the encapsulation of doxorubicin and PFP gas by means of a simple agitation method. The potential of DLMBs for use in theranostics were evaluated under both in vitro and in vivo conditions for cancer therapy and US imaging.

\section{Materials and methods}

\subsection{Phospholipids}

DSPG, 1,2-dipalmitoyl-sn-glycero-3- phosphatidylcholine (DPPC) and PEG 2000 -DSPE were purchased from Avanti Polar Lipid Inc. (Alabaster, AL, USA) and NOF Co. (Tokyo, Japan).

\subsection{Cells}

The B16BL6 murine melanoma cell line was obtained from the American Type Culture Collection (ATCC, Manassas, VA, USA). Cells were cultured in Dulbecco's modified eagle medium Nissui Pharmaceutical Co., Ltd., (Tokyo, Japan) supplemented with 10\% fetal bovine serum and $100 \mathrm{U} / \mathrm{ml}$ penicillin/streptomycin at $37^{\circ} \mathrm{C}$ in $5 \% \mathrm{CO}_{2}$.

\subsection{Animals and tumor models}


Female 6-week-old C57BL6 mice were purchased from the Shizouka Agricultural Cooperation Association for Laboratory Animals (Shizouka, Japan) and female 6-week-old HR1 hairless mice were obtained from Sankyo Laboratory Service Corporation, Inc. (Tokyo, Japan). For preparing tumor bearing mice, $1 \times 10^{6}$ cells in phosphate buffered saline were injected subcutaneously into the left flanks of mice with a 26-gauge needle. Experiments were initiated when tumors reached 5-10 mm in diameter after 9-14 days. All experiments were carried out in accordance with the principles of laboratory animal care in accordance with the Principles of the National Institute of Health and Guidelines for Animal Experiments of Kyoto University; all experiments were approved by Teikyo University School of Medicine Animal Ethics Committee, reference number of approval was: 14-027.

\subsection{Doxorubicin liposome and DLMBs preparation}

DPPC, DSPG and PEG 2000-DSPE in a 7:2.5:0.5 molar ratio were dissolved in chloroform, followed by evaporation of the chloroform in a rotary evaporator at $25^{\circ} \mathrm{C}$ for $30 \mathrm{~min}$; this was followed by further drying under vacuum at room temperature overnight. Ten mg of lipid film was hydrated with $3 \mathrm{ml}$ of $5 \%$ glucose solution at $65^{\circ} \mathrm{C}$ for 60 min under mild agitation. The final lipid concentration after hydration was adjusted to $3 \mathrm{mg} / \mathrm{ml}$. The glucose solution also contained $2 \mathrm{mg}$ doxorubicin. For preparing MBs $0.5 \mathrm{ml}$ of liposomes were added to a 5ml sterilized vial. The air in the vial was replaced with PFP gas (Takachiho Chemical Industries Co., Tokyo, Japan) and after capping $6 \mathrm{ml}$ of PFP was injected. To obtain the MBs a shaking machine (Ultra Mate 2, Victoria, Australia) was used. Temperature was measured after agitation using a needle type thermometer (Custom Co., Tokyo, Japan). Perfluoropropane content analysis was performed as reported previously [21]. The particle size and zeta potential of the liposomes and MBs were determined using a Zetasizer Nano ZS instrument (Malvern Instruments Ltd., Worcestershire, UK). 


\subsection{Doxorubicin binding efficiency}

To determine the binding efficiency of doxorubicin in DLMBs, a sample consisting of a 0.5-ml DLMB dispersion (1.65 mg lipid and $0.33 \mathrm{mg}$ doxorubicin) was centrifuged at 16,000 $\mathrm{g}$ for $2 \mathrm{~min}$. Then the sample was divided to three fractions: a foaming cake at the top which contained doxorubicin loaded MBs; a pellet at the bottom, which contained liposomes; and in-between a solution containing free doxorubicin. The fractions were collected, and then the concentration in each fraction was determined by measuring doxorubicin fluorescence (480 $\mathrm{nm}$ was used as excitation wavelength and doxorubicin signal was detected at $590 \mathrm{~nm}$ ) (FluoroMax4, Horiba, Ltd., Kyoto, Japan).

\subsection{In vitro echogenicity}

The DLMBs were injected into a beaker filled with $500 \mathrm{ml}$ of degassed distilled water at $37^{\circ} \mathrm{C}$ under magnetic stirring. Ultrasound contrast enhancement was observed using an ultrasonography system (Vevo 2100, Visual Sonics, Inc. Toronto, Canada). For additional investigations of DLMBs destruction by higher energy US burst, an external US probe at an intensity of $\left(2 \mathrm{~W} / \mathrm{cm}^{2}\right)$ was used. The process of US burst was repeated until most of the DLMBs had disappeared.

\subsection{In vitro cellular uptake and anti-proliferative assay}

Cellular uptake in vitro was evaluated using a confocal microscopy (Nikon, Tokyo, Japan). BL6BL6 cells were grown on cover glasses in 24 -well plates ( $3 \times 10^{5}$ cells/well). DLMBs were added to the medium, containing a doxorubicin concentration of $5 \mu \mathrm{g} / \mathrm{ml}$. Then a 6-mm US probe was immersed into the well and US irradiation was performed for $60 \mathrm{~s}$ (2 $\mathrm{MHz} ; 2 \mathrm{~W} / \mathrm{cm}^{2} ; 50 \%$ Duty; $10 \mathrm{~Hz}$ ). US acoustic parameters were selected based on the 
previous DLMBs destructibility experiment. After treatment with DLMBs and US, cells were incubated for only 15 min to minimize doxorubicin uptake by others mechanisms rather than sonoporation [10]. Cells washed with PBS (-) three times and fixed with 4\% paraformaldehyde in PBS (-). Fluorescence from doxorubicin was detected by using $480 \mathrm{~nm}$ as excitation wavelength and $590 \mathrm{~nm}$ for detection. The image was processed using ImageJ software.

As for anti-proliferative assay, B16BL6 cells were seeded in 6 -well plates $\left(3 \times 10^{5}\right.$ cells/well) for $24 \mathrm{~h}$; DLMBs/MBs only (0 doxorubicin) were added at different concentrations. US was applied for 60 s (2 MHz; 2W/cm²; 50\% duty; $10 \mathrm{~Hz})$. After treatment, cells were incubated for $5 \mathrm{~h}$ and then the medium was changed and the cells were incubated again for $24 \mathrm{~h}$. After that the 3-(4,5 sec-dimethylthiazol-2-yl)-2,5-diphenyl tetrazolium bromide (MTT) assay was carried out according to the method as previously reported [22].

\subsection{In vivo doxorubicin content in tumors}

DLMBs (70 $\mu$ g doxorubicin and $330 \mu \mathrm{g}$ lipid) in 5\% glucose were intravenously injected at the volume of $200 \mu \mathrm{l}$ and then immediately tumors were irradiated with US for $60 \mathrm{~s}$. Mice were sacrificed 15 min after DLMBs injection and immediately tumors, hearts and livers were harvested, weighed and preserved at $-80^{\circ} \mathrm{C}$ until analyzed. Doxorubicin was extracted by homogenizing the tumors in a mixture of isopropanol and $1 \mathrm{M} \mathrm{HCl}$ aqueous solution $(1: 1 \mathrm{v} / \mathrm{v})$ and incubation for $1 \mathrm{~h}$ at $4^{\circ} \mathrm{C}$. The samples were then centrifuged at 13,000 $\mathrm{g}$ for $15 \mathrm{~min}$, and the supernatants were recovered for fluorescence detection (FluoroMax4, Horiba, Ltd., Kyoto, Japan). Doxorubicin standard series were prepared in non-treated tumor tissues extracts.

\subsection{In vivo tumor inhibition and imaging}


The first treatment was initiated on the $9^{\text {th }}$ day after tumor transplantation and repeated on the $11^{\text {th }}$ and $13^{\text {th }}$ days. Immediately after DLMBs administration intravenously, the tumor site was irradiated for $60 \mathrm{~s}$ with US (2 MHz; 2W/cm²; 50\% Duty; $10 \mathrm{~Hz}$ ). Tumor volume was measured every 2-3 days using the formula: (major axis $\times$ minor axis $\left.{ }^{2}\right) \times 0.5$.

HR1 hairless mice $(n=3)$ inoculated with B16BL6 melanoma were anesthetized and placed on an imaging pad; temperature, heart rate and breathing were continuously monitored. US imaging was performed as previously described [23]. Briefly, a dedicated small animal high spatial-resolution imagining linear transducer (16 MHz; gain, $25 \mathrm{~dB}$; dynamic range, 50 dB) (Vevo 2100) was used. Subsequently, DLMBs were injected at a dose of $100 \mu \mathrm{l} /$ mouse (300 $\mu \mathrm{g} / \mathrm{ml}$ lipid) and short movies (460 frames) were recorded before and at pre-determined time points after injection. For each time point the US contrast power (i.e. brightness of the picture) in the tumor was calculated so that the decay of the signal over time could be evaluated. Also, to further evaluate the potential of the DLMBs for in vivo imaging a maximum intensity persistence (MIP) analysis was made. In this analysis, the highest intensities from each frame is accumulated into one picture so that the bubble positions are highlighted making it easier to distinguish e.g. blood vessels from the background.

\subsection{Statistical analysis}

All data were analyzed as the mean \pm SEM. Unpaired, the two tailed distribution Student t test was applied and values of $P<0.05$ were considered as being statistically significant.

\section{Results}

\subsection{DLMBs characteristics}


The size of the DLMBs was adjusted by means of the mechanical agitation time of the shaking machine. Agitation for $60 \mathrm{~s}$ was sufficient to produce MBs with an average diameter of $1 \mu \mathrm{m}$ (Table 1). During this procedure, the temperature did not exceed $26.7^{\circ} \mathrm{C}$. The fluorescence microscope images showed spherical MBs in which the doxorubicin signal was observed in the shells (supplementary Fig. 1). PFP gas retention was enhanced by the increase of doxorubicin content from 10 to 82\% (Fig. 1). The time course study showed that with a doxorubicin concentration at 10\%, PFP gas leaked even faster than was the case from MBs that did not contain doxorubicin. However, at levels of $42 \%$ and $82 \%$ doxorubicin enhanced PFP gas retention was evident for at least $30 \mathrm{~min}$ at room temperature relative to both MBs (0 doxorubicin) and the control (saline with PFP). The loaded dose of doxorubicin in DLMBs was adjusted to $497.9 \pm 17.4 \mu \mathrm{g} / \mathrm{ml}(\mathrm{n}=3)$, and $92.5 \%$ of the total doxorubicin was loaded into the DLMBs after total separation from free doxorubicin and pellets (supplementary Fig. 2).

The echogenicity of DLMBs was assessed in vitro after injecting the freshly prepared DLMBs at $37^{\circ} \mathrm{C}$. The brightness mode of ultrasonography showed a high signal from the DLMBs even at 10 min (Fig. 2). The acoustic destructibility study involving the DLMBs demonstrated that US irradiation from 40 to 60 s caused a significant decrease in the ultrasonography video intensity as most of the MBs had been destroyed (Fig. 3).

\subsection{In vitro doxorubicin uptake and MTT assay}

In vitro doxorubicin delivery was investigated in B16BL6 cells using confocal scanning microscopy. The combination of DLMBs with US irradiation (Fig. 4A) showed higher cellular accumulation of doxorubicin after 15 min of treatment as compared with DLMBs in the absence of US irradiation (Fig. 4B). The combination of free doxorubicin with US irradiation did not achieve high levels of doxorubicin (Fig. 4C). 
Unloaded MBs in combination with US irradiation resulted in a decrease in tumor cell viability by about 32.5\%, suggesting that there were cavitation effects associated with unloaded MBs in combination with US irradiation. DLMBs used in the absence of US irradiation resulted in a decrease in cell viability of approximately $27 \%$. In contrast, cell viabilities were significantly reduced after treatment with DLMBs combined with US

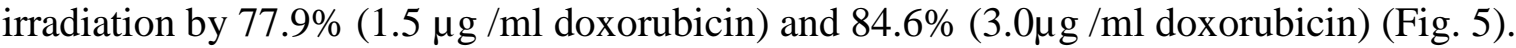

\subsection{Intratumoral content of doxorubicin in tumor bearing mice}

As shown in Fig. 6, the combination of DLMBs with US irradiation significantly enhanced the intra-tumoral doxorubicin level (0.536 $\mu \mathrm{g} / \mathrm{g}$ tissue) as compared with DLMBs without US irradiation (0.100 $\mu \mathrm{g} / \mathrm{g})$ (Fig. 6A). Conversely, the levels of doxorubicin in the heart (Fig. 6B) and the liver (Fig. 6C) did not significantly differ between the two groups.

\subsection{Tumor growth inhibition and body weight change in tumor bearing mice}

By day 27 after tumor transplantation tumor volume in the control group had aggressively increased $\left(7156.2 \pm 1384 \mathrm{~mm}^{3}\right)$. The tumor volume was slightly inhibited relative to the control group (4663.2 $\pm 454 \mathrm{~mm}^{3}$ ) in the group of mice treated with DLMBs in the absence of US irradiation. In contrast, in the group of mice treated with DLMBs in combination with US irradiation there was significant inhibition of tumor volume (2453.9 $\left.\pm 175 \mathrm{~mm}^{3}\right)$ (Fig. 7A). Moreover, treatment with DLMBs in combination with US irradiation did not cause any significant loss of body weight relative to the control group (Fig. 7B).

\subsection{In vivo US imaging}


After $1 \mathrm{~min}$, the intensity of the contrast enhancement signal was gradually reduced (Fig. 8A). The images were analyzed by plotting the mean contrast intensity in the tumor area against time after DLMBs injection (Fig. 8B). We estimated the half-life time of DLMBs to be between 2 and 3 min. Moreover, the maximum intensity persistence (MIP) analysis clearly revealed the distribution of DLMBs in the tumor vasculature with a distinguished contrast signal (Fig. 8C).

\section{Discussion}

Many studies have reported on the different methods by which doxorubicin or paclitaxel was successfully prepared and tested for tumor therapy in combination with US irradiation $[10,24,25]$. Although most of the previous approaches achieved high therapeutic efficacy in vitro and in vivo $[9,26]$, there is a little evidence regarding their potential role in US imaging and whether or not the co-encapsulation of PFP and doxorubicin is effective enough for US contrast enhancement in addition to enhancing the therapeutic potential of doxorubicin.

In this study, we examined DLMBs and studied their potential characteristics regarding both tumor US imaging and therapy. The size of DLMBs was adjusted by means of agitation; initially more agitation lead to smaller MBs and a better distribution, but after $60 \mathrm{~s}$ no further change was observed in the size distribution and the amount of doxorubicin associated with the MBs (data not shown). This result indicates that the majority of doxorubicinphospholipids dispersion would from DLMBs with high doxorubicin loading efficiency (92.5\%). During this procedure, the temperature did not exceed $26.7^{\circ} \mathrm{C}$, suggesting that it is a mild process which could be beneficial if effective compounds could be included in the formulations. The fluorescence images of DLMBs revealed spherical shapes in which doxorubicin was bound to the DSPG in the shell through electrostatic interactions between the positively charged doxorubicin and the negatively charged MBs (data not shown). 
Both doxorubicin loading and PFP gas retention are critical factors for successful theranostic application. We therefore attempted to investigate a possible relationship between PFP gas retention and the amount of doxorubicin intercalated with DSPG in the MBs. Gas chromatography was used to quantify the amount of PFP gas in the DLMBs at different doxorubicin to DSPG ratios. As shown in Fig. 1, a higher doxorubicin loading enhanced PFP gas retention in the DLMBs. These observations led us to believe that not only DSPG incorporation in MBs but also the intercalation between DSPG and doxorubicin is an important factor regarding stable PFP encapsulation.

US burst for a period of $60 \mathrm{~s}$ at an intensity of only $2 \mathrm{~W} / \mathrm{cm}^{2}$ was sufficient to induce a significant decrease in the contrast signal of DLMBs; the presumption was that destruction of the DLMBs most likely occurred as a result of bubble cavitation (Fig. 3). The US burst caused the bubble destruction may be concluded by comparison between Fig. 3 and Fig. 2. As shown in Fig. 2, the same DLMBs monitored with lower energy US but without burst and it shows that bubbles remain after $10 \mathrm{~min}$ whereas the $2 \mathrm{~W} / \mathrm{cm}^{2}$ burst destroyed virtually all bubbles in $40 \mathrm{~s}$. Moreover, we showed that doxorubicin uptake in cancer cells was enhanced when DLMBs were combined with US irradiation relative to free doxorubicin in combination with US irradiation (Fig. 4). Similarly, high in vitro cytotoxicity and effective in vivo delivery to the tumor were achieved.

It is expected that US irradiation at the tumor site leads to DLMBs cavitation and doxorubicin release; consequently, the concentration of free doxorubicin may increase in tumor but not in the heart and liver. Furthermore, DLMBs in combination with US irradiation significantly inhibited tumor growth without any reduction in animal body weight. These results suggest that DLMBs combined with US irradiation at the tumor site improve treatment accuracy and safety with less systemic side effects including cardiotoxicity. 
As shown in Fig. 8, the imaging potentials of DLMBs were confirmed by US echo imaging, which revealed a high contrast enhancement signal in the tumor area in mice after DLMBs administration. Moreover, we showed that the DLMBs contrast signal in the tumor vasculature could be clearly distinguished from the surrounding normal tissues. Pysz et al. have reported that in vivo the ultrasound imaging signal in tumor bearing mice using MIP algorithm could be used to assess tumor vascularity [27]. That is because a correlation between the in vivo MIP values with microvessel density analysis was observed. Therefore, tumor angiogenesis could be monitored by evaluating the MIP analysis of DLMBs distribution in the tumor vasculature. A future study following on from the findings of this study will evaluate modification of MBs with ligands such peptides or antibodies to enable more selective targeting of tumors.

\section{Conclusion}

We succeeded in preparing a novel DLMBs formulation using a simple mechanical agitation method. Doxorubicin bound to DSPG enhanced MB stability relative to unloaded MBs. The in vitro experiments revealed that the uptake of doxorubicin was increased by the combination of DLMBs and US irradiation as compared with the free form of doxorubicin. In mice, doxorubicin distribution in tumor was enhanced when DLMBs were combined with US irradiation at the tumor site. Additionally, US echo imaging showed high contrast enhancement of the DLMBs in the tumor vasculature. These findings suggest that the DLMBs that we prepared achieve US triggered delivery of doxorubicin as well as US imaging of tumor for theranostics. These observations could provide valuable information for the design rationale for DLMBs concerning cancer theranostics.

\section{Acknowledgments}


This work was supported in part by a Grant-in-Aid for Scientific Research on Innovative Areas from the Ministry of Education, Culture, Sports, Science and Technology of Japan, by the Programs for Promotion of Fundamental Studies in Health Sciences of the National Institute of Biomedical Innovation (NIBIO) and by the Uehara Memorial Foundation.

\section{References}

[1] Hashida M, Kawakami S, Yamashita F. Lipid Carrier Systems for Targeted Drug and Gene Delivery. Chem Pharm Bull (Tokyo) 2005;53:871-80.

[2] Jain RK, Stylianopoulos T. Delivering nanomedicine to solid tumors. Nat Rev Clin Oncol 2010;7:653-64.

[3] Lindner JR, Song J, Xu F, Klibanov AL, Singbartl K, Ley K, et al. Noninvasive ultrasound imaging of inflammation using microbubbles targeted to activated leukocytes. Circulation 2000;102:2745-50.

[4] Van Wamel A, Kooiman K, Harteveld M, Emmer M, ten Cate FJ, Versluis M, et al. Vibrating microbubbles poking individual cells: drug transfer into cells via sonoporation. J Control Release 2006;112:149-55.

[5] Deng CX, Sieling F, Pan H, Cui J. Ultrasound-induced membrane porosity. Ultrasound Med \\& Biol 2004;30:519-26.

[6] Karshafian R, Bevan PD, Williams R, Samac S, Burns PN. Sonoporation by ultrasound-activated microbubble contrast agents: effect of acoustic exposure parameters on cell membrane permeability and cell viability. Ultrasound Med Biol 2009;35:847-60.

[7] Niu C, Wang Z, Lu G, Krupka TM, Sun Y, You Y, et al. Doxorubicin loaded superparamagnetic PLGA-iron oxide multifunctional microbubbles for dual-mode US/MR imaging and therapy of metastasis in lymph nodes. Biomaterials 2013;34:2307-17.

[8] Korpanty G, Carbon JG, Grayburn PA, Fleming JB, Brekken RA. Monitoring response to anticancer therapy by targeting microbubbles to tumor vasculature. Clin Cancer Res 2007;13:323-30.

[9] Ueno Y, Sonoda S, Suzuki R, Yokouchi M, Kawasoe Y, Tachibana K, et al. Combination of ultrasound and bubble liposome enhance the effect of doxorubicin and inhibit murine osteosarcoma growth. Cancer Biol Ther 2011;12:270-7. 
[10] Lentacker I, Geers B, Demeester J, De Smedt SC, Sanders NN. Design and evaluation of doxorubicin-containing microbubbles for ultrasound-triggered doxorubicin delivery: cytotoxicity and mechanisms involved. Mol Ther 2010;18:101-8.

[11] Goormaghtigh E, Chatelain P, Caspers J, Ruysschaert JM. Evidence of a specific complex between adriamycin and negatively-charged phospholipids. Biochim Biophys Acta 1980;597:1-14.

[12] De Wolf FA. Binding of doxorubicin to cardiolipin as compared to other anionic phospholipids--an evaluation of electrostatic effects. Biosci Rep 1991;11:275-84.

[13] Qin S, Caskey CF, Ferrara KW. Ultrasound contrast microbubbles in imaging and therapy: physical principles and engineering. Phys Med Biol 2009;54:R27-R57.

[14] Suzuki R, Takizawa T, Negishi Y, Hagisawa K, Tanaka K, Sawamura K, et al. Gene delivery by combination of novel liposomal bubbles with perfluoropropane and ultrasound. J Control Release 2007;117:130-6.

[15] Un K, Kawakami S, Suzuki R, Maruyama K, Yamashita F, Hashida M. Development of an ultrasound-responsive and mannose-modified gene carrier for DNA vaccine therapy. Biomaterials 2010;31:7813-26.

[16] Un K, Kawakami S, Yoshida M, Higuchi Y, Suzuki R, Maruyama K, et al. The elucidation of gene transferring mechanism by ultrasound-responsive unmodified and mannose-modified lipoplexes. Biomaterials 2011;32:4659-69.

[17] Un K, Kawakami S, Yoshida M, Higuchi Y, Suzuki R, Maruyama K, et al. Efficient suppression of murine intracellular adhesion molecule-1 using ultrasound-responsive and mannose-modified lipoplexes inhibits acute hepatic inflammation. Hepatology 2012;56:259-69.

[18] Kurosaki T, Kawakami S, Higuchi Y, Suzuki R, Maruyama K, Sasaki H, et al. Development of anionic bubble lipopolyplexes for efficient and safe gene transfection with ultrasound exposure in mice. J Control Release 2014;176:24-34.

[19] Kurosaki T, Kawakami S, Higuchi Y, Suzuki R, Maruyama K, Sasaki H, et al. Kidneyselective gene transfection using anionic bubble lipopolyplexes with renal ultrasound irradiation in mice. Nanomedicine 2014.

[20] Sax N, Kodama T. Optimization of acoustic liposomes for improved in vitro and in vivo stability. Pharm Res 2013;30:218-24.

[21] Shiraishi K, Endoh R, Furuhata H, Nishihara M, Suzuki R, Maruyama K, et al. A facile preparation method of a PFC-containing nano-sized emulsion for theranostics of solid tumors. Int J Pharm 2011;421:379-87.

[22] Ogawara K, Un K, Tanaka K, Higaki K, Kimura T. In vivo anti-tumor effect of PEG liposomal doxorubicin (DOX) in DOX-resistant tumor-bearing mice: Involvement of cytotoxic effect on vascular endothelial cells. J Control Release 2009;133:4-10. 
[23] Negishi Y, Hamano N, Tsunoda Y, Oda Y, Choijamts B, Endo-Takahashi Y, et al. AG73-modified Bubble liposomes for targeted ultrasound imaging of tumor neovasculature. Biomaterials 2013;34:501-7.

[24] Fan C-H, Ting C-Y, Lin H-J, Wang C-H, Liu H-L, Yen T-C, et al. SPIO-conjugated, doxorubicin-loaded microbubbles for concurrent MRI and focused-ultrasound enhanced brain-tumor drug delivery. Biomaterials 2013;34:3706-15.

[25] Yan F, Li X, Jin Q, Jiang C, Zhang Z, Ling T, et al. Therapeutic ultrasonic microbubbles carrying paclitaxel and LyP-1 peptide: preparation, characterization and application to ultrasound-assisted chemotherapy in breast cancer cells. Ultrasound Med Biol 2011;37:768-79.

[26] Tinkov S, Coester C, Serba S, Geis NA, Katus HA, Winter G, et al. New doxorubicinloaded phospholipid microbubbles for targeted tumor therapy: in-vivo characterization. J Control Release 2010;148:368-72.

[27] Pysz MA, Foygel K, Panje CM, Needles A, Tian L, Willmann JK. Assessment and monitoring tumor vascularity with contrast-enhanced ultrasound maximum intensity persistence imaging. Invest Radiol 2011;46:187-95.

\section{Disclosure}

The authors declare that there is no conflict of interests. 
Fig. 1. Time course study of DLMBs with different doxorubicin concentrations presented as percentage equivalent to anionic phospholipids. Mean \pm SEM. ${ }^{*} P<0.05$ versus the corresponding control group (saline with PFP gas).

Fig. 2. DLMBs echogenicity in vitro. (A) US imaging experimental set up. (B) Images of DLMBs in brightness mode.

Fig. 3. In vitro DLMBs acoustic destructibility. After the injection of DLMBs US echo imaging was performed and then the US destruction beam was applied from the external probe at an intensity of $2 \mathrm{~W} / \mathrm{cm}^{2}$. (A) Images of DLMBs brightness and contrast mode after the application of US irradiation. (B) Graph presenting DLMBs contrast signal regression after 6 volumes of US irradiation (10 s each).

Fig. 4. Confocal laser microscopy images. (A) DLMBs in combination with US irradiation, (B) DLMBs in the absence of US irradiation and (C) free doxorubicin in combination with US irradiation (scale bar, $50 \mu \mathrm{m}$ ). Cells were treated with DLMBs and free doxorubicin in combination with US irradiation and then incubated for 15 min prior to the analysis.

Fig. 5. Viability of B16BL6 melanoma cells after treatment with DLMBs, unloaded microbubbles (0 doxorubicin) and US irradiation alone. Each bar represents the mean \pm SEM of 6 experiments. $* P<0.05$ versus the corresponding groups with no treatment (N.T.).

Fig. 6. Doxorubicin distribution characteristics after intravenous administration of DLMBs with or without US irradiation in tumor bearing mice. The doxorubicin content was measured in (A) tumor, (B) heart and (C) liver. Each bar represents the mean \pm SEM of five 
experiments. $* P<0.05$ versus the corresponding group that received DLMBs in combination with US irradiation.

Fig. 7. Tumor inhibition study. (A) Tumor volume in mice. (B) Mouse body weight. Mice were divided into three groups: control; DLMBs only; and DLMBs in combination with US irradiation. Treatment was performed on the $9^{\text {th }}$ day after tumor transplantation and was repeated on the on the $11^{\text {th }}$ and $13^{\text {th }}$ days. Each bar represents the mean \pm SEM of 5-6 experiments. $* P<0.05$

Fig. 8. Ultrasound imaging of DLMBs in tumors. (A) Ultrasound images of DLMB signal enhancement in both brightness mode (left) and contrast mode (right). (B) Time-intensity graph for DLMBs at the tumor site after intravenous administration. (C) MIP analysis images of DLMBs distribution in the tumor vasculature after intravenous injection.

Supplementary Fig. 1. Fluorescence images based doxorubicin detection. (scale bar of 10 $\mu \mathrm{m})$.

Supplementary Fig. 2. DLMBs separation through buoyancy effects after centrifugation. (1) DLMBs (2) free doxorubicin (3) pellets of phospholipids aggregates with doxorubicin.

Table 1. Mean particle size and zeta potential of liposomes and microbubbles. $n=3$; mean \pm SEM.

\begin{tabular}{|l|c|c|}
\hline & $\begin{array}{c}\text { Mean particle size } \\
(\mathrm{nm})\end{array}$ & $\begin{array}{c}\text { Zeta potential } \\
(\mathrm{mV})\end{array}$ \\
\hline Unloaded liposome & $250 \pm 1$ & $-0.076 \pm 0.02$ \\
\hline DOX loaded liposome & $270 \pm 6$ & $0.038 \pm 0.02$ \\
\hline Unloaded microbubbles (MBs) & $1051 \pm 4$ & $-0.066 \pm 0.04$ \\
\hline DOX loaded microbubbles (DLMBs) & $1022 \pm 5$ & $0.031 \pm 0.01$ \\
\hline
\end{tabular}


Fig. 1

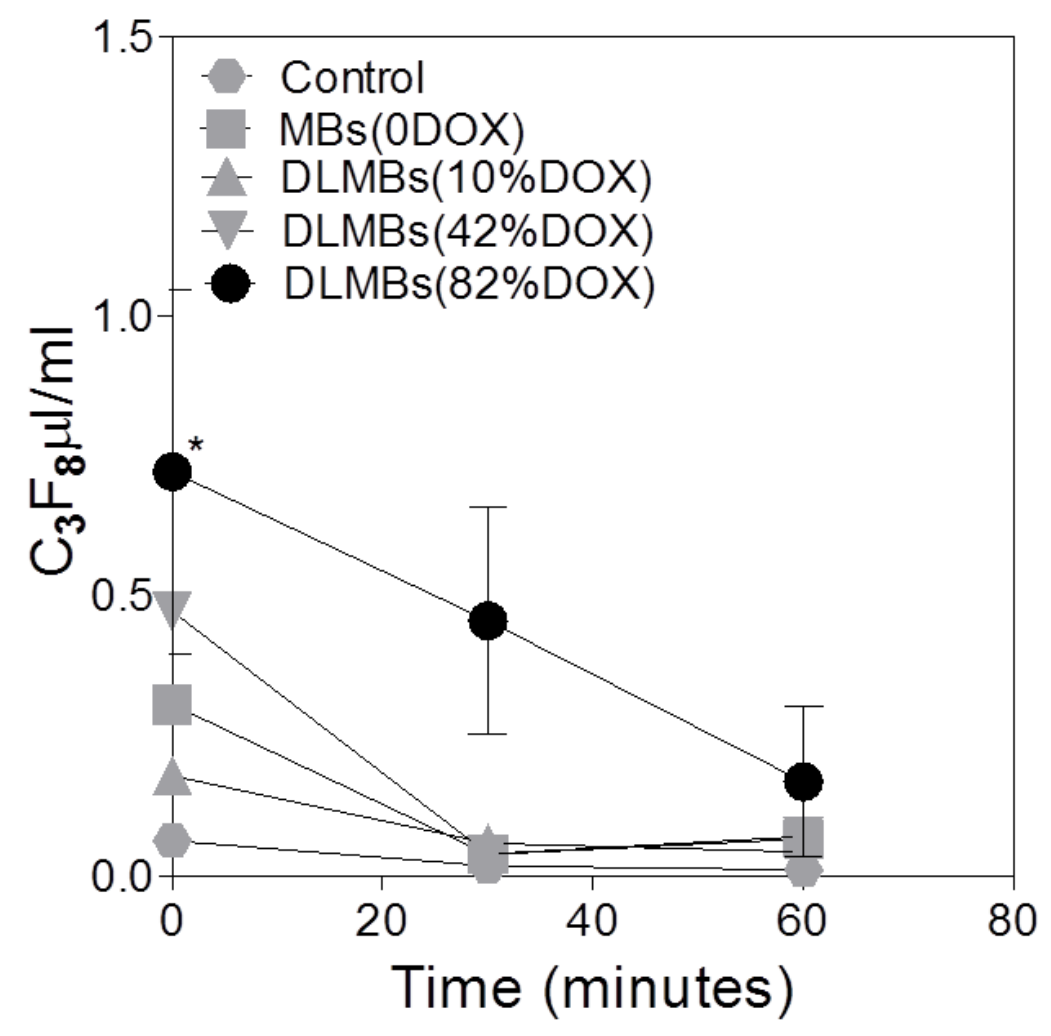


Fig. 2
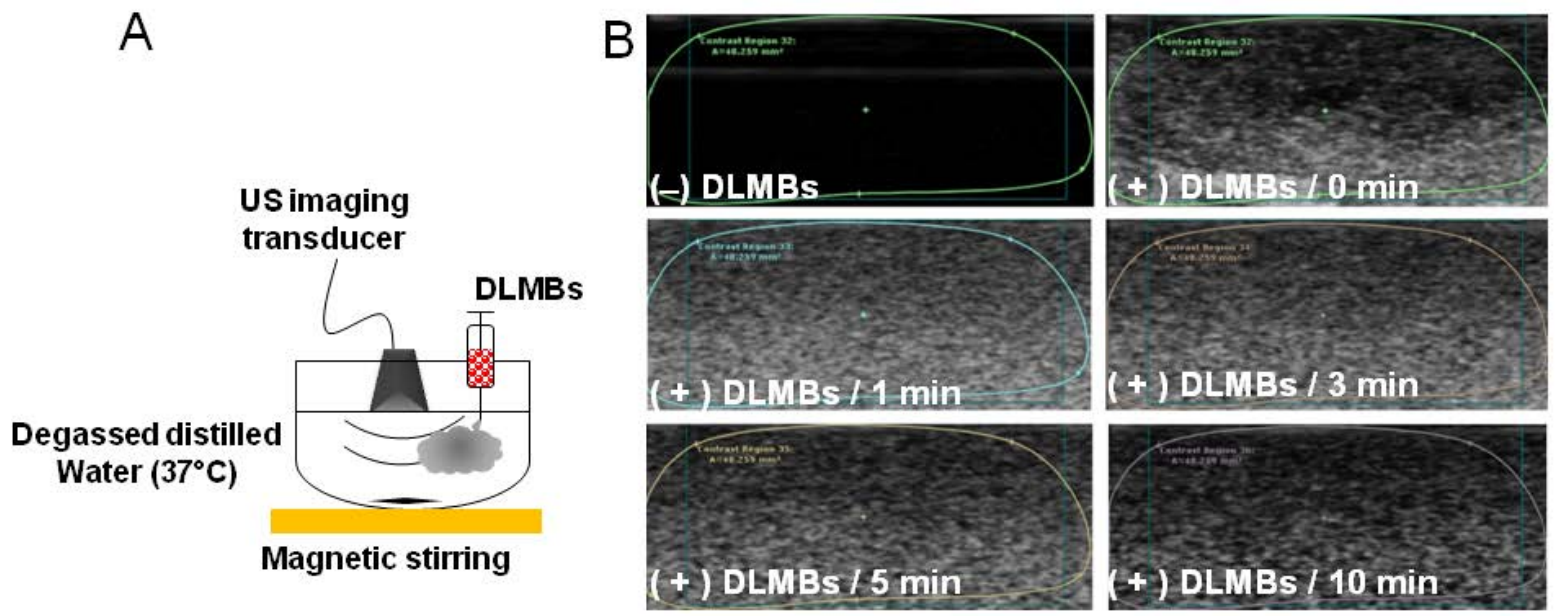
Fig. 3
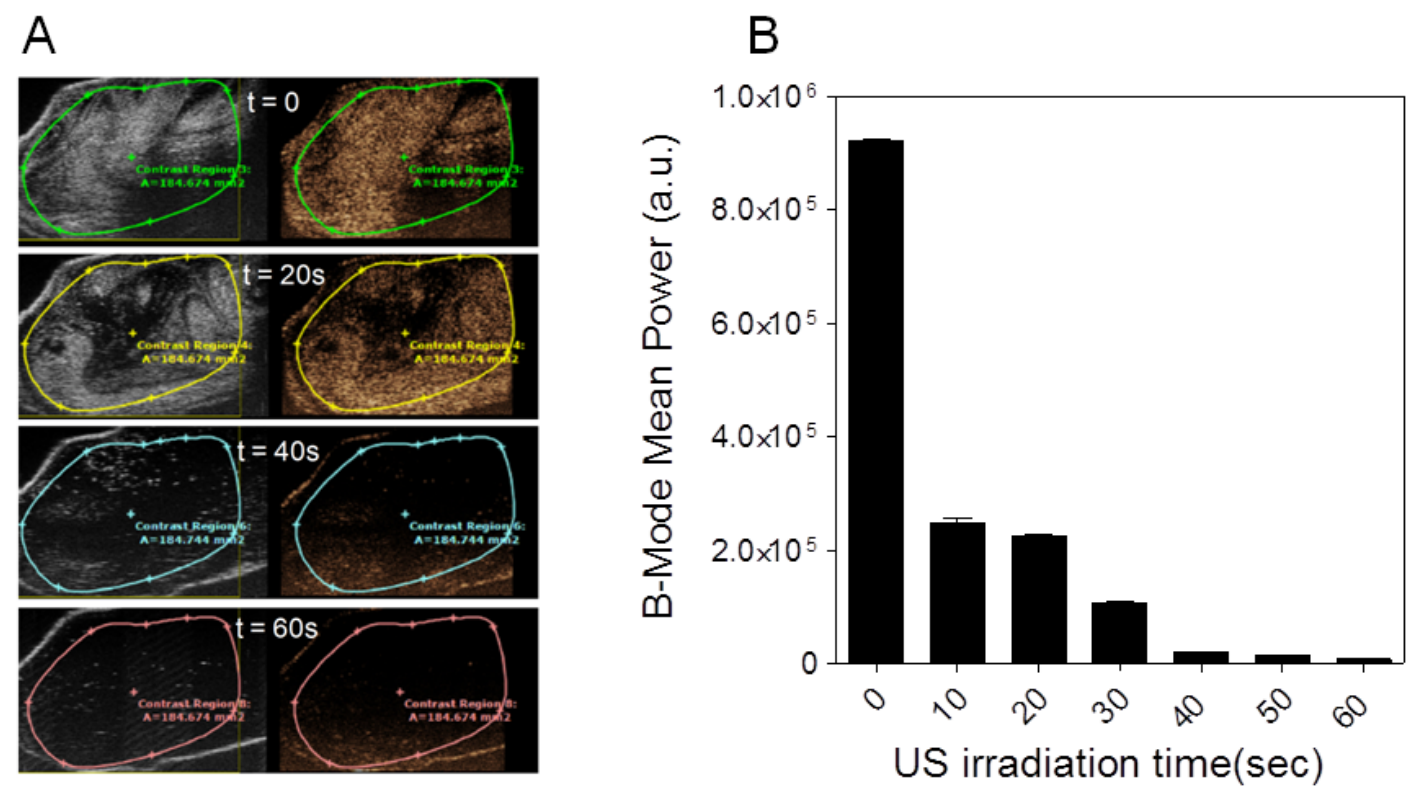
Fig. 4

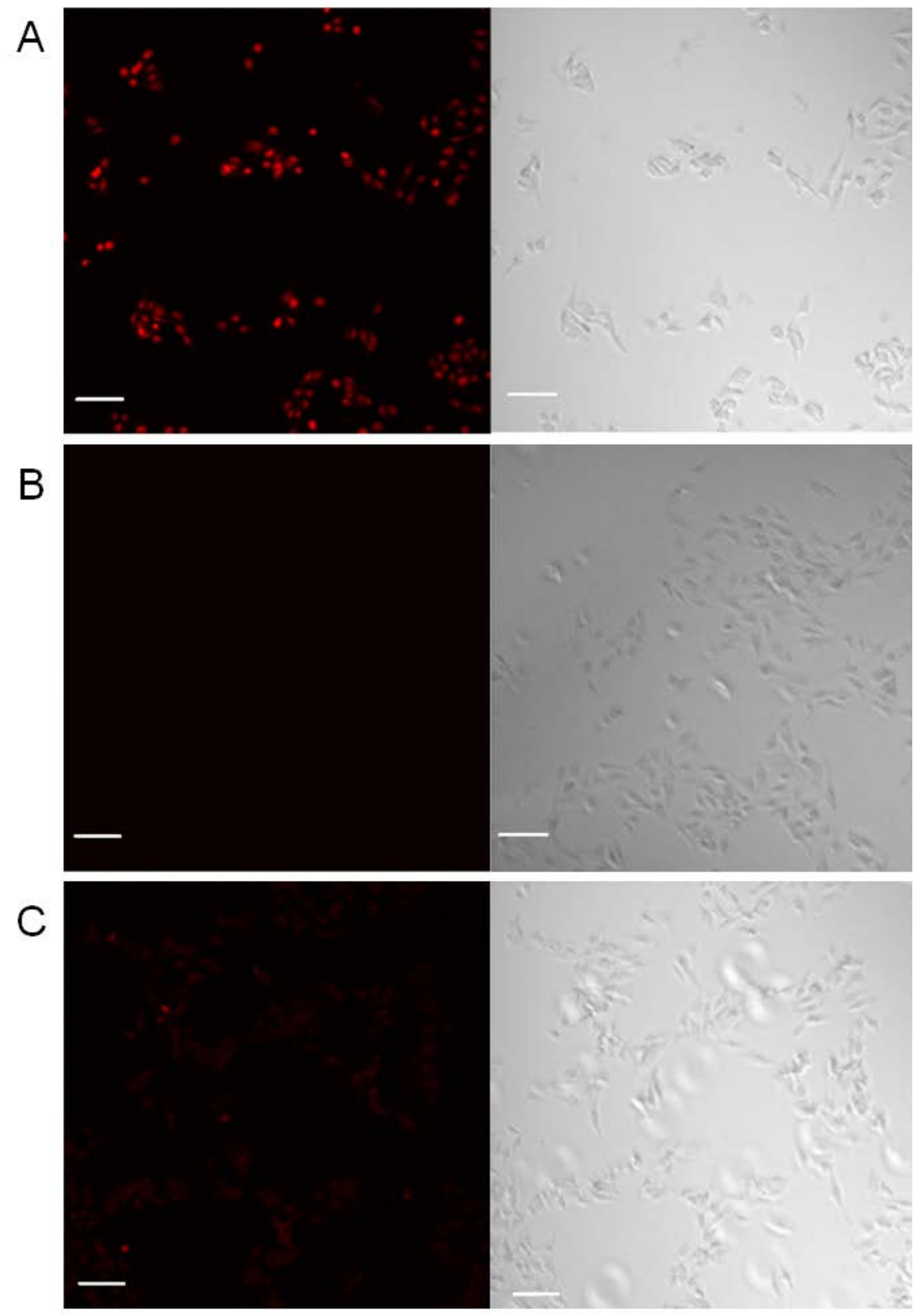


Fig. 5

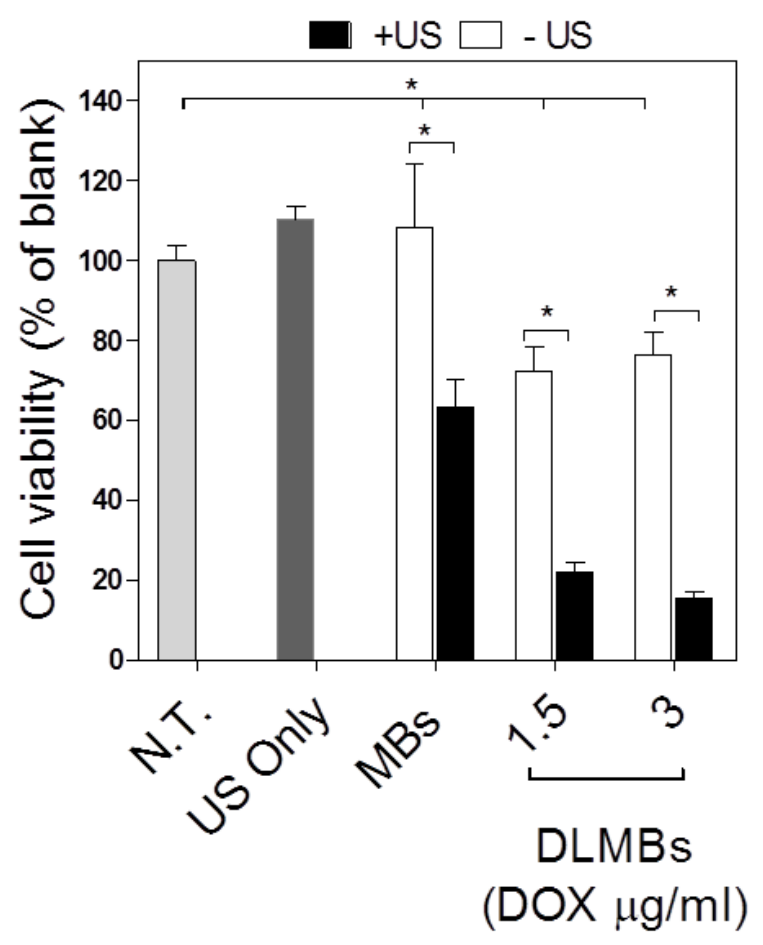


Fig. 6
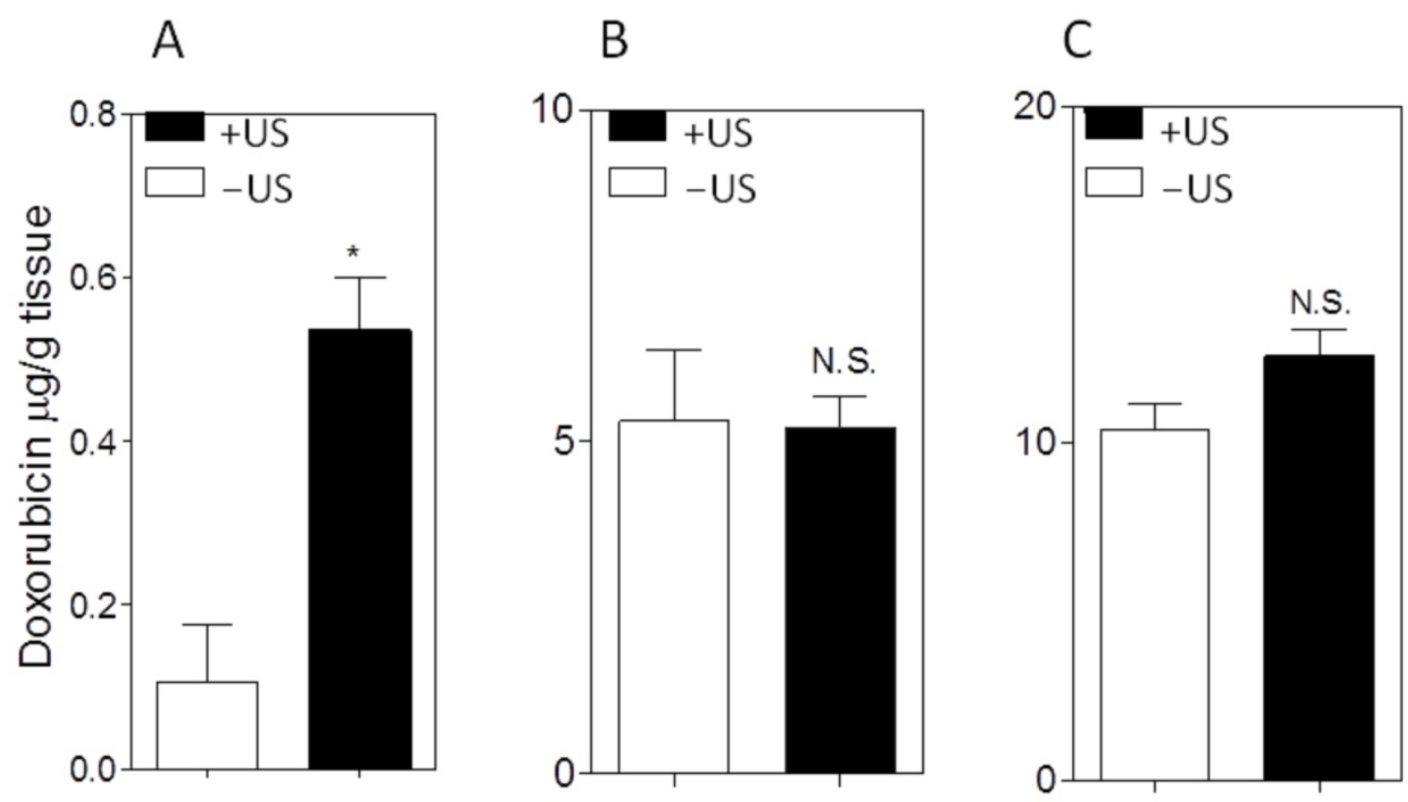
Fig. 7
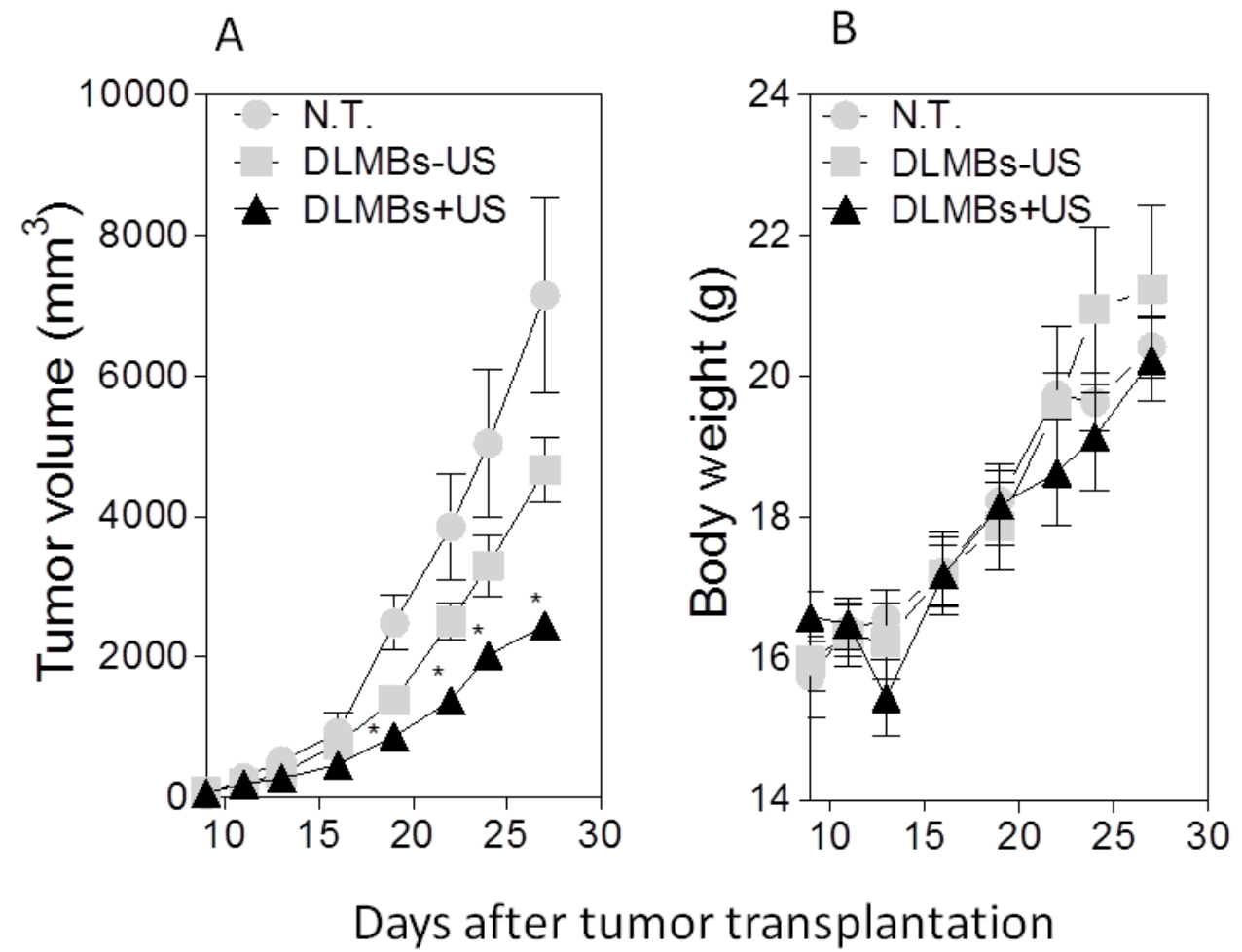
Fig. 8
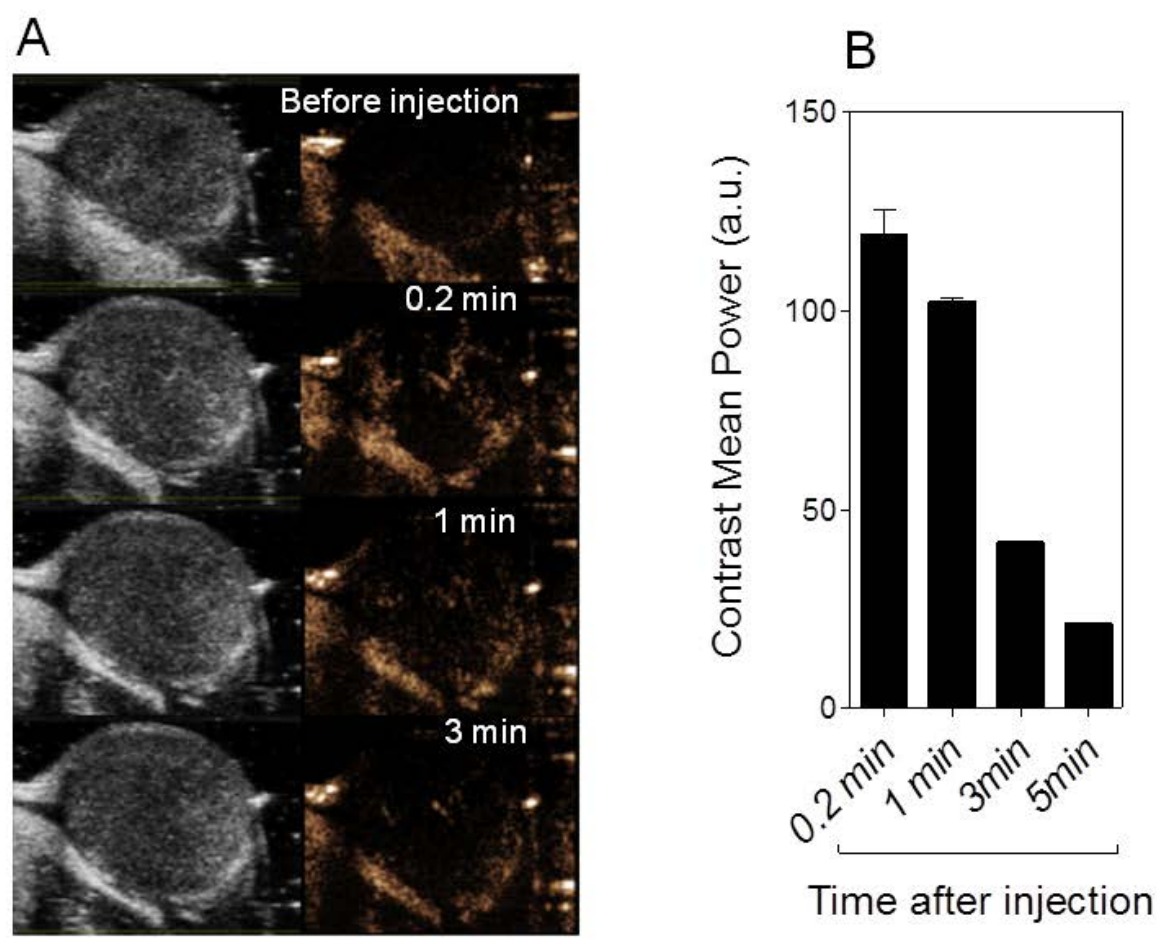

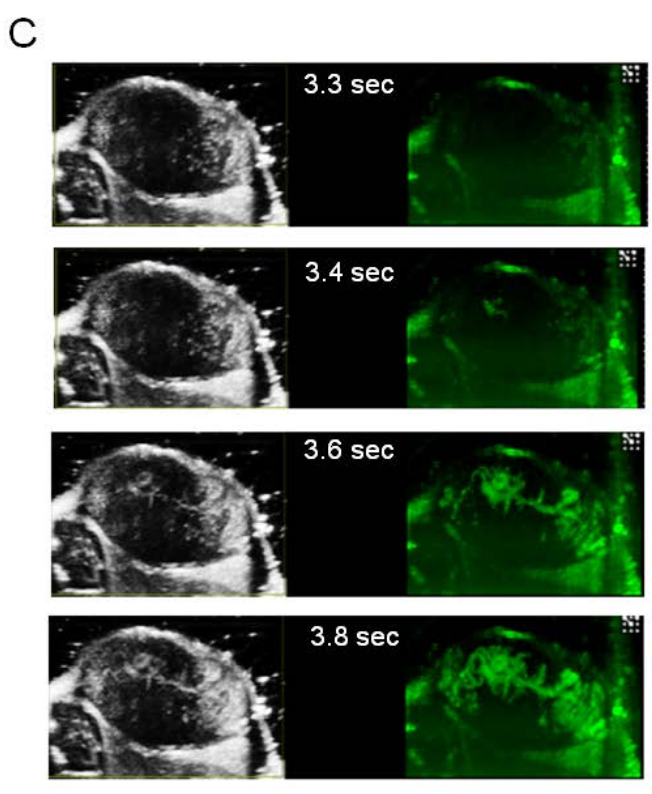
Supplementary Fig. 1

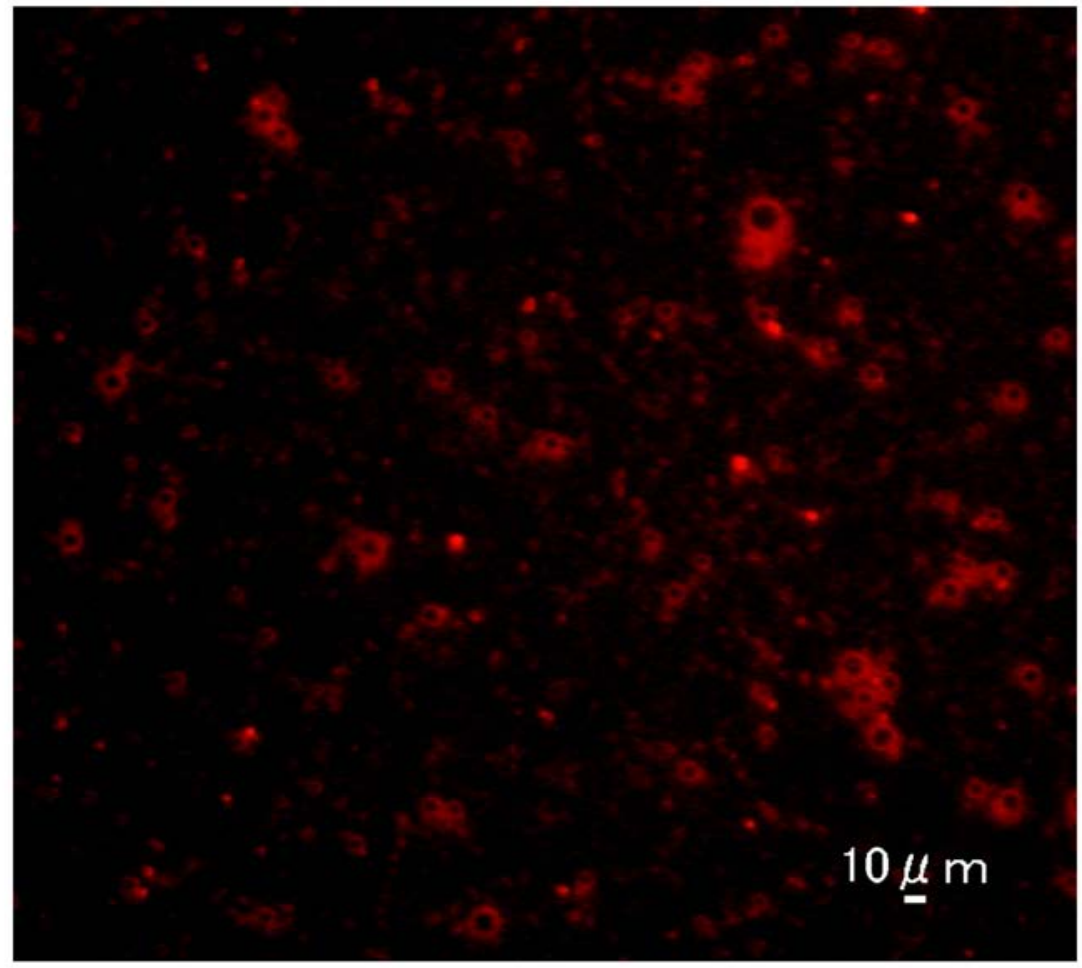


Supplementary Fig. 2

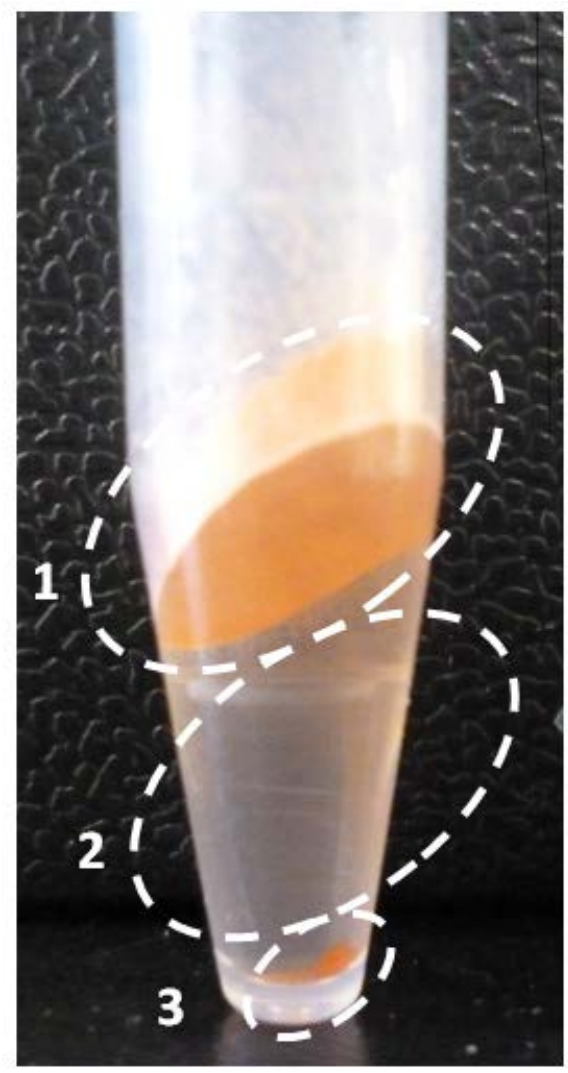

